\title{
About time nondeterminism and exception handling in a temporal extension of LOTOS*
}

\author{
Jean-Pierre Courtiat and Roberto C. de Oliveira ${ }^{\dagger}$ \\ LAAS-CNRS, 7 avenue du Colonel Roche, 31077 Toulouse Cedex, France \\ e-mail: $\{$ courtiat,cruz $\} @$ laas.fr
}

\begin{abstract}
The paper deals with the study of a temporal extension of Basic LOTOS, and addresses in particular issues related to the expression of time nondeterminism and to the specification of temporal exception handling behaviors. The underlying framework of the proposed extension stems from our previous proposal, RT-LOTOS, and from the work conducted by G. Leduc on Timed LOTOS and, more recently on ET-LOTOS. Main contribution of our proposal is related to the introduction of a new operator, the so-called latency operator, which provides a simple and elegant solution for expressing time nondeterminism. As far as temporal exception handling is concerned, we assess different potential solutions, from the simplest based on the use of the choice operator to the more complex (from a semantical point of view) based on the temporal disrupt operator, initially introduced in RT-LOTOS. For reason of convergence with the work of G. Leduc, we use, as much as possible, syntactic constructs initially defined for Timed LOTOS and ET-LOTOS. The paper presents also some results about temporal bisimulations, and shows in particular that the proposed weak bisimulation is a congruence for the hide and the enabling operators.
\end{abstract}

Keyword Codes: C.2.4; D.2.1; F.4.3

Keywords: Distributed Systems; Requirement/Specifications; Formal Languages

\section{Introduction}

In this paper we study several temporal extensions of the formal description technique Basic LOTOS [BB87] with the purpose of providing a minimal set of powerful temporal operators able to express the temporal requirements commonly encountered in the formal specification of real-time systems and applications.

When designing a formal language or an extension to a formal language, one is faced with different design requirements, which sometimes may appear to be in contradiction the one with the others. For instance, it is desirable:

1. to limit as much as possible the number of constructs (i.e. the number of operators in a process algebra)

2. to design constructs that may be easily formalized (i.e. whose semantics is as simple as possible)

3. to guarantee that each construct has a clearly identified purpose

4. to have constructs which emphasize a structured style of specification

*This work has been supported by CNET (Grant 92-1B-178) as part of CESAME, a CNET-CNRS collaborative project on High Speed Multimedia Systems

†Partly supported by a grant from $\mathrm{CNPq} / \mathrm{Brazil}$ 
5. to have also high-level constructs available which, although not mandatory, may be very interesting when using the formal language for specifying complex systems

The paper tries to provide answers to these issues, as far as temporal extensions of LOTOS are concerned. For this reason, we show progressively how our proposal may be elaborated starting from a very simple model which is then step by step refined. This makes it possible to provide a better understanding of the basic semantic rules, to study their relationships, as well as to propose, at some stage, alternative ways to express a particular feature.

Initial inspiration for this work came from an in-depth analysis of the temporal extension proposed by G. Leduc, namely Timed LOTOS as presented in [LL92], as well as of the one proposed by T. Bolognesi, namely T-LOTOS as presented in [BL92]. In particular, the debate between Timed LOTOS and T-LOTOS has generated a discussion on whether or not observable actions are to be urgent. In [CdCS93], we proposed our own temporal extension of basic LOTOS, namely RT-LOTOS, which has been somehow inspired by the aforementioned debate. In RT-LOTOS, we have chosen as a starting point that observable actions are not urgent due to the fact that their occurrence depends on the willingness of their environment (like in Timed LOTOS), but also that these actions cannot be performed outside their specific time interval; hence, the idea to introduce the temporal violation concept, leading naturally to the temporal disrupt operator. In [LL93a], and presented at the same conference as RT-LOTOS, G. Leduc has proposed an Extended Timed LOTOS (called ET-LOTOS in the sequel), where the time during which an observable action may be offered is also limited, as in RT-LOTOS. However, some main differences between RT-LOTOS and ET-LOTOS remain, and are essentially related to the ways:

1. temporal constraints are associated with the actions

2. actions are being offered

3. time non determinism is dealt with

4. temporal exception handling is treated

The purpose of this paper is therefore, by elaborating step by step a new proposal, to conciliate as much as possible the interesting features of both Timed LOTOS (and ET-LOTOS) and RT-LOTOS, and to propose solutions for some problems which were still pending in ET-LOTOS and RT-LOTOS, trying to meet as much as possible the different design requirements stated previously.

The paper is organized as follows: Section 2 introduces the two main models which are considered in this paper and shows precisely how they are related; the first model is the basic model which refers only to a deterministic delay operator, whereas the second model extends the previous one by the introduction of the so-called latency operator for expressing time nondeterminism. Section 3 proposes and assesses several ways for expressing temporal exception handling to take place when some (observable) actions cannot fulfill their temporal requirements. Section 4 introduces a strong and a weak temporal bisimulations and shows some useful properties. Conclusions are finally drawn in section 5 .

\section{The models}

\subsection{Background assumptions}

\subsubsection{Time domain}

We consider exactly the same assumptions as the ones made for Timed LOTOS, ET-LOTOS and RTLOTOS. The unique assumption made is that the time domain is countable, which implies that the underlying semantics model is indeed a LTS (Labeled Transition System). Proceeding in this way implies that we can consider a discrete time domain (natural integers), as well as a dense time domain (rational numbers) [NS91].

Let $D$ be the time domain. $D^{\infty}$ denotes $D \cup\{\omega\}$ where $\omega \stackrel{\text { def }}{\notin} D$ and $\forall t \in D, t<\omega$ and $\omega+t=\omega$.

We will use $t, u, v$ to range over $D^{\infty}$. It will be further assumed that $t>0$ in all tables of the operational semantics presented in the paper. 


\subsubsection{Notations}

Let $A$ be the alphabet of observable actions, $i$ the internal action and $\delta$ the termination action. The following sets of actions are then defined:

- $A^{i}=A \cup\{i\}$

- $A^{\delta}=A \cup\{\delta\}$

- $A^{i, \delta}=A \cup\{i, \delta\}$

The following notations will also be used for expressing the inference rules:

- $P \stackrel{a}{\rightarrow} P^{\prime}$ means that process $P$ may realize action $a$ and behaves then like process $P^{\prime}$

- $P \stackrel{a}{\rightarrow}$ means that $\exists P^{\prime}$ such that $P \stackrel{a}{\rightarrow} P^{\prime}$

- $P \stackrel{a}{\dagger}$ means that process $P$ cannot realize action $a$

- $P \stackrel{t}{\rightarrow} P^{\prime}$ with $t \in D^{\infty}$, means process $P$ may idle (i.e. it does not execute any action) during a period of $t$ units of time and behaves after like process $P^{\prime}$.

\subsection{The basic model}

\subsubsection{Motivations}

An important issue to be dealt with is related to the way an action is being offered, once enabled. Three main approaches may be considered:

1. a "punctual" offering at the instant the action becomes enabled

2. a "punctual" offering at any time during a time interval, either bounded or not

3. a "continuous" offering during a time interval, either bounded or not

Some formalisms have taken different approaches depending on whether the action is observable or internal (remember furthermore that the internal action can be introduced either directly by prefixing or by the hiding of another action).

In Timed-LOTOS and ET-LOTOS, approach 3 has been applied to the observable actions and approach 1 is valid for the internal action. In Timed-LOTOS, the time interval considered in approach 3 is unbounded by definition, whereas it may be bounded in ET-LOTOS. Moreover, in ET-LOTOS, an additional construct, namely $i\{t\}$, can be used in a prefix such that to satisfy approach 2 .

In RT-LOTOS, approach 2 (with a bounded or an unbounded time interval) has been used for both the observable actions and the internal action, which implies that the RT-LOTOS model, as detailed in [CdCS93], does not feature the maximal progress property. As a consequence, the interpretation of behavior [ $\mathrm{t} 1, \mathrm{t} 2] \mathrm{a} ; \mathrm{P}$ in RT-LOTOS is rather different from behavior $\Delta^{t 1} a\{t 2-t 1\} ; P$ in ET-LOTOS, as the RT-LOTOS behavior introduces some kind of additional internal time nondeterminism.

It is our belief that time nondeterminism issues should be clearly separated from delay issues and therefore expressed by a distinct construct. This is the case for ET-LOTOS by means of the $i\{t\}$ construct and of the $\Delta^{t}$ operator, but not for RT-LOTOS, as presented in [CdCS93]. However, we will see in the next paragraph that the $i\{t\}$ construct is not completely satisfactory, and that a more elegant solution of time nondeterminism is provided by the so-called latency operator. 


\begin{tabular}{|c|c|c|}
\hline$(1 . b)$ & exit $\stackrel{\delta}{\rightarrow}$ stop & $\begin{array}{l}\text { (1.a) } \text { stop } \stackrel{t}{\rightarrow} \text { stop } \\
\text { (1.c) exit } \stackrel{t}{\rightarrow} \text { exit } \\
\end{array}$ \\
\hline$(2 . a)$ & $a\{u\} ; P \stackrel{a}{\rightarrow} P(a \in A)$ & $\begin{array}{l}(2 . b) \quad a\{u+t\} ; P \stackrel{t}{\rightarrow} a\{u\} ; P(a \in A) \\
(2 . c) \quad a\{0\} ; P \stackrel{t}{\rightarrow} \text { stop }(a \in A)\end{array}$ \\
\hline$(3 . a)$ & $i ; P \stackrel{i}{\rightarrow} P$ & \\
\hline$(4 . a)$ & $\frac{P \stackrel{a}{\rightarrow} P^{\prime}}{\Delta^{0} P \stackrel{a}{\rightarrow} P^{\prime}}\left(a \in A^{i, \delta}\right)$ & $\begin{array}{l}\text { (4.b) } \Delta^{u+t} P \stackrel{t}{\rightarrow} \Delta^{u} P \\
\text { (4.c) } \frac{P \stackrel{t}{\rightarrow} P^{\prime}}{\Delta^{0} P \stackrel{t}{\rightarrow} P^{\prime}}\end{array}$ \\
\hline (5.a) & $\frac{P \stackrel{a}{\rightarrow} P^{\prime}}{P[] Q \stackrel{a}{\rightarrow} P^{\prime}}\left(a \in A^{i, \delta}\right)$ & (5.b) $\frac{P \stackrel{t}{\rightarrow} P^{\prime} Q \stackrel{t}{\rightarrow} Q^{\prime}}{P[] Q \stackrel{t}{\rightarrow} P^{\prime}[] Q^{\prime}}$ \\
\hline $\begin{array}{l}(6 . a) \\
(6 . c)\end{array}$ & $\begin{array}{c}\frac{P \stackrel{a}{\rightarrow} P^{\prime} \stackrel{a}{Q} \stackrel{a}{\rightarrow} Q^{\prime}}{P|[L]| Q \stackrel{a}{\rightarrow} P^{\prime}|[L]| Q^{\prime}}(a \in L \cup\{\delta\}) \\
\frac{P \stackrel{a}{\rightarrow} P^{\prime}}{P|[L]| Q \stackrel{a}{\rightarrow} P^{\prime}|[L]| Q}\left(a \in A^{i} \backslash L\right)\end{array}$ & (6.b) $\frac{P \stackrel{t}{\rightarrow} P^{\prime} Q \stackrel{t}{\rightarrow} Q^{\prime}}{P|[L]| Q \stackrel{t}{\rightarrow} P^{\prime}|[L]| Q^{\prime}}$ \\
\hline $\begin{array}{l}(7 . a) \\
(7 . c)\end{array}$ & $\begin{array}{l}\frac{P \stackrel{a}{\rightarrow} P^{\prime}}{\text { hide } L \text { in } P \stackrel{a}{\rightarrow} \text { hide } L \text { in } P^{\prime}}\left(a \in A^{i, \delta} \backslash L\right) \\
\frac{P \stackrel{a}{\rightarrow} P^{\prime}}{\text { hide } L \text { in } P \stackrel{i}{\rightarrow} \text { hide } L \text { in } P^{\prime}}(a \in L)\end{array}$ & (7.b) $\frac{P \stackrel{t}{\rightarrow} P^{\prime} P \stackrel{a}{\rightarrow} \quad \forall a \in L}{\text { hide } L \text { in } P \stackrel{t}{\rightarrow} \text { hide } L \text { in } P^{\prime}}$ \\
\hline $\begin{array}{l}(8 . a) \\
(8 . c)\end{array}$ & $\begin{array}{l}\frac{P \stackrel{a}{\rightarrow} P^{\prime}}{P \gg Q \stackrel{a}{\rightarrow} P^{\prime} \gg Q}\left(a \in A^{i}\right) \\
P \stackrel{\delta}{\rightarrow} P^{\prime} \\
P \gg Q \stackrel{i}{\rightarrow} Q\end{array}$ & (8.b) $\frac{P \stackrel{t}{\rightarrow} P^{\prime} P \stackrel{\delta}{\oplus}}{P \gg Q \stackrel{t}{\rightarrow} P^{\prime} \gg Q}$ \\
\hline $\begin{array}{l}(9 . a) \\
(9 . c) \\
(9 . d)\end{array}$ & $\begin{array}{l}\frac{P \stackrel{a}{\rightarrow} P^{\prime}}{P\left[>Q \stackrel{a}{\rightarrow} P^{\prime}[>Q\right.}\left(a \in A^{i}\right) \\
\frac{Q \stackrel{a}{\rightarrow} Q^{\prime}}{P\left[>Q \stackrel{a}{\rightarrow} Q^{\prime}\right.}\left(a \in A^{i, \delta}\right) \\
\frac{\stackrel{5}{\rightarrow} P^{\prime}}{P\left[>Q \stackrel{\circ}{\rightarrow} P^{\prime}\right.}\end{array}$ & (9.b) $\frac{P \stackrel{t}{\rightarrow} P^{\prime} Q \stackrel{t}{\rightarrow} Q^{\prime}}{P\left[>Q \stackrel{t}{\rightarrow} P^{\prime}\left[>Q^{\prime}\right.\right.}$ \\
\hline$(10 . a)$ & $\frac{E\left[a_{1} / a_{1}^{\prime} \cdots a_{n} / a_{n}^{\prime}\right] \stackrel{g}{\rightarrow} E^{\prime} \quad P\left[a_{1}^{\prime} \cdots a_{n}^{\prime}\right]:=E}{P\left[a_{1} \cdots a_{n}\right] \stackrel{g}{\rightarrow} E^{\prime}}$ & $\left(g \in A^{i, \delta} \cup D^{\infty}\right)$ \\
\hline
\end{tabular}

Table 1: The basic model 


\subsubsection{Operational semantics}

For the previous reason, our basic model will not feature any time nondeterminism capability. For reasons of convergence with G. Leduc's work, and because a delay operator may appear to be more adequate for ensuring a structured style of specification (see design criterion 4), we use operator $\Delta^{t}$ instead of associating a temporal label directly with the actions. Notation $a\{t\}$ is also used for expressing a continuous offering of observable action $a$ during a $t$ time period, but construct $i\{t\}$ is not supported as previously mentioned.

The operational semantics of the basic model is provided in table 1, in which the symmetric rules have been omitted. It is almost straightforward and may be seen as being derived from both [LL93a, LL93b].

\subsubsection{Assessment}

The main shortcoming of this basic model is that it is not able to express time nondeterminism. This issue is addressed in the next paragraph by means of the introduction of the latency operator. The definition of two operators, the deterministic delay operator and the latency operator, instead of an unique operator $\Delta^{t, u} P$ like in Timed-LOTOS, make it possible to clearly separate the delay and the time nondeterminism issues, satisfying therefore design criterion 3 . The proposed solution will also be shown to be more general than the $i\{t\}$ construct of ET-LOTOS.

\subsection{Adding time nondeterminism in the basic model}

\subsubsection{Motivations}

This model extends the basic model by adding time nondeterminism. The deterministic behavior of the basic model results from the deterministic delay operator and from the urgent nature of the three following types of actions:

- observable actions are urgent when hidden

- termination action $\delta$ is urgent when appearing at the left of $>>$ operator

- internal action $i$ is urgent in the classical meaning $(P \stackrel{i}{\rightarrow} \Rightarrow \forall t \neq 0, P \stackrel{t}{\oplus})$

The following example illustrates this purely deterministic behavior. Let process $\mathrm{S}$ be defined as: $\mathrm{S}$ $=$ hide a in ( $\Delta^{t}$ a; $\left.\mathrm{P}|[\mathrm{a}]| \Delta^{u} \mathrm{a} ; \mathrm{Q}\right)$; then, action $a$ will occur deterministically at instant $\max (t, u)$ due to the urgency of the interactions among hidden observable actions.

In order to express time nondeterminism, we propose to revise the three notions of urgency defined in the basic model. For this purpose, two "semantical" actions, noted $a_{s}$ and $a_{w}$, are associated with each action $a \in A^{i, \delta}$ of the syntactical model. $a_{s}$, which will be called a strong a action, features the same urgency characteristics as action $a$ in the basic model; $a_{w}$, which will be called a weak $a$ action, features a non-urgent behavior in the three situations considered above, i.e.:

- $a_{w}$ with $a \in$ Act is not urgent when hidden

- $\delta_{w}$ is not urgent when appearing at the left of operator $\gg$

- $i_{w}$ is not urgent

In order to generate a weak semantical action from its corresponding action in the syntactical model, a new operator, the latency operator $\Omega^{t}$, is defined. When applying $\Omega^{t}$ to some process $P$, the first action possibly offered by $P$ during the time interval of duration $t$, is interpreted as a weak semantical action. At the last instant of the time interval, operator $\Omega^{t}$ is no more active (as the time interval it defines is open at its right). $\Omega^{t}$ actuates only on the first action offered by process $P$, which means that it actuates only on either the actions of $P$ which are already enabled or the actions of $P$ which will become enabled when process $P$ idles.

\subsubsection{Operational semantics}

The complete formal semantics is provided in table 2 , in which rules (1.b), (2.a), (6.a), (7.b), (7.c), (8.b), (8.c) and (10.a) are an adaptation of the similar rules in Table 1 and rules $\left(4^{\prime}{ }^{*}\right)$ express the semantics of 


\begin{tabular}{|c|c|c|}
\hline$(1 . b)$ & exit $\stackrel{\delta_{s}}{\rightarrow}$ stop & $\begin{array}{l}(1 . a) \text { stop } \stackrel{t}{\rightarrow} \text { stop } \\
(1 . c) \text { exit } \stackrel{t}{\rightarrow} \text { exit }\end{array}$ \\
\hline$(2 . a)$ & $a\{u\} ; P \stackrel{a_{a}}{\rightarrow} P(a \in A)$ & $\begin{array}{l}(2 . b) \quad a\{u+t\} ; P \stackrel{t}{\rightarrow} a\{u\} ; P(a \in A) \\
(2 . c) \quad a\{0\} ; P \stackrel{t}{\rightarrow} \text { stop }(a \in A)\end{array}$ \\
\hline $\begin{array}{l}(3 . a) \\
(3 . c)\end{array}$ & $\begin{array}{l}i\{t\} ; P \stackrel{i_{w}}{\rightarrow} P \\
i\{0\} ; P \stackrel{i_{s}}{\rightarrow} P\end{array}$ & $(3 . b) \quad i\{u+t\} ; P \stackrel{t}{\rightarrow} i\{u\} ; P$ \\
\hline$(4 . a)$ & $\frac{P \stackrel{a}{\rightarrow} P^{\prime}}{\Delta^{0} P \stackrel{a}{\rightarrow} P^{\prime}}\left(a \in A^{i, \delta}\right)$ & $\begin{array}{l}\text { (4.b) } \Delta^{u+t} P \stackrel{t}{\rightarrow} \Delta^{u} P \\
\text { (4.c) } \frac{P \stackrel{t}{\rightarrow} P^{\prime}}{\Delta^{0} P \stackrel{t}{\rightarrow} P^{\prime}}\end{array}$ \\
\hline $\begin{array}{l}\left(4^{\prime} . a\right) \\
\left(4^{\prime} . c\right) \\
\left(4^{\prime} . e\right)\end{array}$ & $\begin{array}{l}\frac{P \stackrel{a}{\rightarrow} P^{\prime}}{\Omega^{t} P \stackrel{a_{w}}{\rightarrow} P^{\prime}}\left(a \in A^{\delta}\right) \\
\frac{P \stackrel{i}{\rightarrow} P^{\prime}}{\Omega^{t} P \stackrel{i}{\rightarrow} P^{\prime}} \\
\frac{P \stackrel{a}{\rightarrow} P^{\prime}}{\Omega^{0} P \stackrel{a}{\rightarrow} P^{\prime}}\left(a \in A^{i, \delta}\right)\end{array}$ & $\begin{array}{l}\left(4^{\prime} . b\right) \frac{P \stackrel{t}{\rightarrow} P^{\prime}}{\Omega^{u+t} P \stackrel{t}{\rightarrow} \Omega^{u} P^{\prime}} \\
\left(4^{\prime} . d\right) \frac{P \stackrel{t}{\rightarrow} P^{\prime}}{\Omega^{0} P \stackrel{t}{\rightarrow} P^{\prime}}\end{array}$ \\
\hline$(5 . a)$ & $\frac{P \stackrel{a}{\rightarrow} P^{\prime}}{P[] Q \stackrel{a}{\rightarrow} P^{\prime}}\left(a \in A^{i, \delta}\right)$ & (5.b) $\frac{P \stackrel{t}{\rightarrow} P^{\prime} Q \stackrel{t}{\rightarrow} Q^{\prime}}{P[] Q \stackrel{t}{\rightarrow} P^{\prime}[] Q^{\prime}}$ \\
\hline$(6 . a)$ & 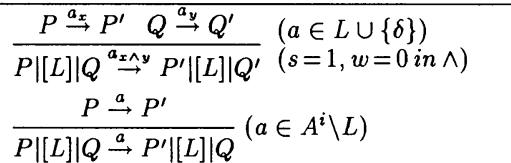 & (6.b) $\frac{P \stackrel{t}{\rightarrow} P^{\prime} Q \stackrel{t}{\rightarrow} Q^{\prime}}{P|[L]| Q \stackrel{t}{\rightarrow} P^{\prime}|[L]| Q^{\prime}}$ \\
\hline $\begin{array}{l}(7 . a) \\
(7 . c)\end{array}$ & $\begin{array}{l}\frac{P \stackrel{a}{\rightarrow} P^{\prime}}{\text { hide } L \text { in } P \stackrel{a}{\rightarrow} \text { hide } L \text { in } P^{\prime}}\left(a \in A^{i, \delta} \backslash L\right) \\
\frac{P \stackrel{a_{x}}{\rightarrow} P^{\prime}}{\text { hide } L \text { in } P \stackrel{i_{x}}{\rightarrow} \text { hide } L \text { in } P^{\prime}}(a \in L)\end{array}$ & (7.b) $\frac{P \stackrel{t}{\rightarrow} P^{\prime} P \stackrel{a}{\rightarrow} \quad \forall a \in L}{\text { hide } L \text { in } P \stackrel{t}{\rightarrow} \text { hide } L \text { in } P^{\prime}}$ \\
\hline $\begin{array}{l}(8 . a) \\
(8 . c)\end{array}$ & $\begin{array}{l}\frac{P \stackrel{a}{\rightarrow} P^{\prime}}{P \gg Q \stackrel{a}{\rightarrow} P^{\prime} \gg Q}\left(a \in A^{i}\right) \\
P \stackrel{\delta_{x}}{\rightarrow} P^{\prime} \\
P \gg Q \stackrel{i_{x}}{\rightarrow} Q\end{array}$ & (8.b) $\frac{P \stackrel{t}{\rightarrow} P^{\prime} \quad P^{\delta_{\dot{H}}}}{P \gg Q}$ \\
\hline $\begin{array}{l}(9 . a) \\
(9 . c) \\
(9 . d)\end{array}$ & $\begin{array}{l}\frac{P \stackrel{a}{\rightarrow} P^{\prime}}{P\left[>Q \stackrel{a}{\rightarrow} P^{\prime}[>Q\right.}\left(a \in A^{i}\right) \\
\frac{Q \stackrel{a}{\rightarrow} Q^{\prime}}{P\left[>Q \stackrel{a}{\rightarrow} Q^{\prime}\right.}\left(a \in A^{i, \delta}\right) \\
\frac{P \stackrel{\delta}{\rightarrow} P^{\prime}}{P\left[>Q \stackrel{\delta}{\rightarrow} P^{\prime}\right.}\end{array}$ & (9.b) $\frac{P \stackrel{t}{\rightarrow} P^{\prime} Q \stackrel{t}{\rightarrow} Q^{\prime}}{P\left[>Q \stackrel{t}{\rightarrow} P^{\prime}\left[>Q^{\prime}\right.\right.}$ \\
\hline$(10 . a)$ & $\frac{E\left[a_{1} / a_{1}^{\prime} \cdots a_{n} / a_{n}^{\prime}\right] \stackrel{g}{\rightarrow} E^{\prime} \quad P\left[a_{1}^{\prime} \cdots a_{n}^{\prime}\right]:=E}{P\left[a_{1} \cdots a_{n}\right] \stackrel{g}{\rightarrow} E^{\prime}}$ & $\left(g \in\left\{a_{s}, a_{w} \mid a \in A^{i, \delta}\right\} \cup D^{\infty}\right)$ \\
\hline
\end{tabular}

Table 2: The basic model with time nondeterminism 
operator $\Omega^{t}$. Some explanations of these rules are provided in the sequel, and note that the symmetric rules have been omitted:

1. $a_{x}$ stands for either $a_{s}$ or $a_{w}$, when it is required to make explicit in the inference rules the type (strong or weak) of the semantical actions being considered

2. $a$ stands for either $a_{s}$ or $a_{w}$, when there is no need in the inference rules for expliciting the type of the semantical actions being considered

3. rules (1.b), (2.a) and (3.c) characterize the occurrence of strong semantical actions associated respectively with a termination action, an observable action and an internal action

4. rule (3.a) characterizes the occurrence of a weak internal action

5. rule (4'.a) shows how strong and weak observable actions transform themselves into weak actions; this is the basic purpose of the latency operator; note, by considering rule (4'.c), that the latency operator does not affect internal actions; rule (4'.e) states that the latency operator is without any effect when its time period is equal to zero

6. rules (4'.b) and (4'.d) are the time progression rules for the latency operator

7. rule (6.a) characterizes the synchronization of strong and weak $a$ actions; note that synchronizing a strong action with a weak action leads to a weak action

8. rule (7.b), together with rule (7.c), expresses that hiding strong observable actions leads to the strong internal action, $i_{s}$, which is urgent; on the contrary, hiding weak observable actions leads to the weak internal action, $i_{w}$, which is not urgent

9. rule (8.b), together with rule (8.c), characterizes the urgency of the termination of a process, which depends on whether the termination action is strong or weak

To illustrate further the relationship between strong and weak actions, and to see how weak actions are useful for expressing time nondeterminism, let us consider the two following processes:

$\mathrm{S} 1$ = hide $\mathrm{a}$ in $(\mathrm{a} ; \mathrm{P})$ and $\mathrm{S} 2(\mathrm{~d})=$ hide $\mathrm{a}$ in $\left(\Omega^{d} \mathrm{a} ; \mathrm{P}\right)$ whose specific behaviors may be defined as follows:

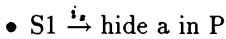

- $\mathrm{S} 1 \stackrel{t}{\dagger}$ for all $t>0$

- $\mathrm{S} 2(\mathrm{~d}) \stackrel{i_{w}}{\longrightarrow}$ hide a in $\mathrm{P}$, if $d>0$

- $\mathrm{S} 2(\mathrm{~d}) \stackrel{t}{\rightarrow}$ hide a in $\left(\Omega^{d-t}\right.$ a; $\left.\mathrm{P}\right)$, for $0<t \leq d$

- $\mathrm{S} 2(0) \stackrel{i}{\rightarrow}$ hide a in $\mathrm{P}$

- $\mathrm{S} 2(0) \stackrel{t}{\nrightarrow}$ for all $t>0$

Finally, looking at the rules of both tables 1 and 2, one may note that all the actions in table 1 are implicitly considered as being strong actions. Rules (4'.a) and (6.a) of table 2 are the two basic rules making possible to transform a strong action into the corresponding weak action. Note furthermore that construct $i\{t\}$ has been introduced in table 2, although not necessary in our model, only for comparison purpose with the equivalent construct of ET-LOTOS. 


\subsubsection{Properties}

The model features the following temporal properties.

Proposition 1 (maximal progress)

If $\mathrm{P} \stackrel{i_{s}}{\rightarrow} \mathrm{P}^{\prime}$ for some $\mathrm{P}^{\prime}$, then, for all $t>0, P \stackrel{t}{\dagger}$.

Proposition 2 (time determinacy)

If $\mathrm{P} \stackrel{t}{\rightarrow} \mathrm{P}^{\prime}$ and $\mathrm{P} \stackrel{t}{\rightarrow} \mathrm{P}^{\prime}$, then $\mathrm{P}^{\prime}$ and $\mathrm{P}^{\prime}$ are identical.

Proposition 3 (time continuity)

For all $t$ and $u$, if $\mathrm{P}^{t+u} \rightarrow \mathrm{P}^{\prime}$ then there exists $\mathrm{P}^{\prime}$ such that $\mathrm{P} \stackrel{t}{\rightarrow} \mathrm{P}^{\prime} \stackrel{u}{\rightarrow} \mathrm{P}$.

\subsubsection{Assessment}

The purpose here is to assess the latency operator with respect to the $i\{t\}$ construct introduced in ETLOTOS for expressing time nondeterminism. The main problem with the $i\{t\}$ construct is that it resolves the choice, whereas $\Omega^{\mathrm{t}}$ does not. Then the question: is this problem really marginal as pointed out in [LL93b] ?

To see that the problem is not really marginal, let us consider the following example, which consists of the specification of a communication medium featuring a transmission delay whose value belongs to some interval $[\mathrm{m}, \mathrm{M}]$. Let a be the action corresponding to the emission of some message across the medium, and $\mathrm{b}$ the action corresponding to the reception of that message after a non-deterministic transmission delay. Action error characterizes the error situation when the environment is not ready to receive the message delivered by the transmission medium; it is further assumed that the medium becomes unavailable (i.e. behaves as stop) as soon as an error occurs. The temporal pattern associated with the occurrence of the actions is presented in figure 1 .

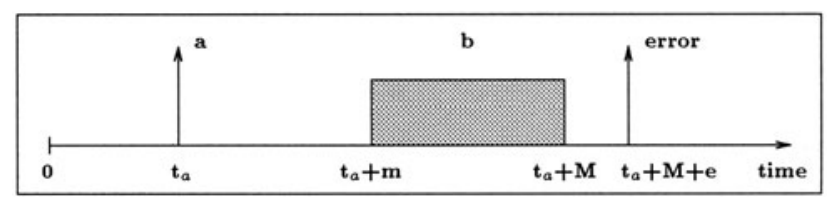

Figure 1: Temporal pattern for offering $b$ action

Using the latency operator, the specification may be expressed as follows:

Medium $=\mathrm{a} ;\left(\Delta^{\mathrm{m}} \Omega^{\mathrm{M}-\mathrm{m}} \mathrm{b}\{\mathrm{M}-\mathrm{m}\} ;\right.$ Medium [ ] $\Delta^{M+e}$ error; stop $)$

Using construct $i\{t\}$ instead of operator $\Omega^{\mathrm{t}}$, what the specification of process Medium would be? The specification below is not correct as $i\{t\}$ resolves the choice.

Medium $=\mathrm{a} ;\left(\Delta^{\mathrm{m}} \mathrm{i}\{\mathrm{M}-\mathrm{m}\} ; \mathrm{b}\{0\} ;\right.$ Medium [ ] $\Delta^{M+e}$ error; stop $)$

We might then think in using the disrupt operator instead of the choice operator for escaping from the previous problem, leading to the following specification:

Medium $=\mathrm{a} ;\left(\Delta^{\mathrm{m}} \mathrm{i}\{\mathrm{M}-\mathrm{m}\} ; \mathrm{b}\{0\} ; \operatorname{Medium}\left[>\Delta^{M+e}\right.\right.$ error; stop $)$

It may easily be seen that this specification is not correct, as action error may occur after an occurrence of action $b$.

The following specifications are alternatives which seem to exhibit the same behavior as the initial specification based on the $\Omega^{\mathrm{t}}$ operator. 


$$
\begin{aligned}
& \text { Medium }=\mathrm{a} ;\left(\Delta^{\mathrm{m}} \mathrm{i}\{\mathrm{M}-\mathrm{m}\} ; \mathrm{b}\{0\} ; \text { exit }\left[>\Delta^{M+e} \text { error; stop }\right)>>\right.\text { Medium } \\
& \text { Medium }=\mathrm{a} ; \Delta^{\mathrm{m}}\left((\mathrm{i}\{\mathrm{M}-\mathrm{m}\} ;(\mathrm{b}\{0\} ; \text { exit [] exit }))|[\mathrm{b}]|\left(\mathrm{b} ; \text { exit [ ] } \Delta^{M-m+e} \text { error; stop }\right)\right)>\text { Medium } \\
& \text { Medịum }=\mathrm{a} ; \Delta^{\mathrm{m}}((\mathrm{i}\{\mathrm{M}-\mathrm{m}\} ;(\mathrm{b} ; \text { exit [ ] exit }))|[\mathrm{b}]|(\mathrm{b}\{\mathrm{M}-\mathrm{m}\} ; \text { exit [ ] exit })|[\mathrm{b}]| \\
& \text { (b; exit [] } \Delta^{M-m+e} \text { error; stop) ) } \gg \text { Medium }
\end{aligned}
$$

The two first specifications, which are simpler than the third one, may cause some problems when combined with another process imposing additional temporal constraints on the occurrence of the $b$ action. The third specification seems to correspond to the initial specification (up to some weak equivalence), but this has not been formally checked.

\section{Temporal exception handling}

By means of construct $a\{t\}$, we have seen that the offering of some particular observable action $a$ may be limited in time. The following question arises: what should be done when the temporal requirement associated with $a$ cannot be satisfied by its particular environment? Such a situation will be qualified as a temporal violation of action $a\{t\}$.

The operational semantics proposed so far provides a very simple answer to this question (see inference rule (2.c)), as the process prefixed by $a\{t\}$ transforms itself in stop.

However, what is really required for the specification of real-time systems is to be able to express, as completely and easily as possible, some temporal exception mechanism to be executed as soon as a temporal violation situation occurs.

\subsection{Motivations}

Our aim is therefore to assess whether the time model presented so far has the capability of expressing satisfactorily temporal exception mechanisms, and, in case of a negative conclusion, to propose additional features for this purpose.

To understand better the issues behind a temporal violation situation, let us analyze the following simple example:

$\mathrm{P}=a\{0\} ; \mathrm{P}^{\prime}$

Let furthermore $\mathrm{E}$ be the particular environment of $\mathrm{P}$, then the complete system specification can be modeled by:

$\mathrm{S}=$ hide $a$ in $(\mathrm{P}|[a]| \mathrm{E})$

Let $\mathrm{Q}$ be the exception process (it is obviously assumed that behavior $\mathrm{Q}$ does not appear in the specification of process P) to be executed in case of a temporal violation of action $a$, then the following behaviors are expected depending on the actions offered by $\mathrm{E}$ at the current time:

1. if $\mathrm{E} \stackrel{a_{a}}{\rightarrow} \mathrm{E}$, then action $a$ should occur, and consequently none of the actions of $\mathrm{Q}$ is allowed to occur

2. if $\mathrm{E} \stackrel{a_{w}}{\rightarrow} \mathrm{E}$, then either action $a$ or an action of $\mathrm{Q}$ may occur

3. if $\mathrm{E} \stackrel{a}{\leftrightarrow} \mathrm{E}$ ' and $\mathrm{E} \stackrel{a_{w}}{\leftrightarrow} \mathrm{E}$, then an action of $\mathrm{Q}$ should occur

\subsection{Use of the choice operator}

A natural way to introduce exception behavior $\mathrm{Q}$ in our specification consists in using the choice operator. Let therefore process $\mathrm{P} 1$ be defined as follows:

$\mathrm{P} 1=a\{0\} ; \mathrm{P}^{\prime}[] \mathrm{Q}$

It is furthermore assumed that process $Q$ offers action error_a for indicating that action $a$ of process $P$ has not been executed. This action error_a can basically be offered in two different ways, which are illustrated by the two specifications below (processes Q1 and Q2) of process Q:

1. Q1 $=\Delta^{\epsilon}$ error_a; Q', which indicates that action error_a is offered after some additional (possible low) delay $\epsilon>0$ to be specified explicitly 
2. Q2 = error_a; Q', which indicates that action error_a is offered without any additional delay $\epsilon$

Let us now analyze the behavior of specification $S$ for the two alternatives of process $Q$ in order to check whether this specification meets the previous requirements:

1. $\mathrm{S}=$ hide $a$ in ( $\left.\left(a\{0\} ; \mathrm{P}^{\prime}[] \mathrm{Q} 1\right)|[a]| \mathrm{E}\right)$

$\mathrm{S}$ meets indeed the previous requirements, as it may be shown below taking into account the actions offered by process E:

(a) if $\mathrm{E} \stackrel{a_{3}}{\rightarrow} \mathrm{E}^{\prime}$, then action $a$ is urgent under the hide and $\mathrm{S} \stackrel{i_{s}}{\rightarrow}$ hide $a$ in ( $\mathrm{P}^{\prime}|[a]| \mathrm{E}^{\prime}$ ), moreover, for all $t>0, \mathrm{~S} \stackrel{t}{\leftrightarrow}$, therefore error_a is not allowed to occur

(b) if $\mathrm{E} \stackrel{a_{w}}{\longrightarrow} \mathrm{E}$, then action $a$ is not urgent under the hide so either action $a$ or action error_a may occur

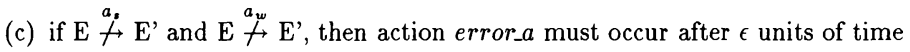

2. $\mathrm{S}=$ hide $a$ in ( $\left.\left(a\{0\} ; \mathrm{P}^{\prime}[] \mathrm{Q} 2\right) \|[a] \mid \mathrm{E}\right)$

In this case, one can easily see that requirements 2 and 3 are met, but that requirement 1 is not satisfied. This is due to the fact that, at time 0 , there exists a nondeterminism between an occurrence of action $a$ and an occurrence of action error_a. Therefore requirement 1 is not satisfied, since $\mathrm{S}$ may realize action error_a, even if environment $\mathrm{E}$ offers action $a_{s}$.

In conclusion of this paragraph, one can say that there exists indeed a solution based on the use of the choice operator for executing an exception treatment in case of a temporal violation situation, but that this solution presents three main shortcomings:

1. the first action of the exception process has to be prefixed by a delay operator with a strictly positive value

2. the choice alternative with the exception process specification (or an instantiation of the exception process) has to be expressed at the same level as the one of the relevant observable action

3. the choice alternative with the exception process specification has to be expressed explicitly for all the occurrences of the relevant observable action

\subsection{Use of a temporal violation action}

In order to address (partially) the previous shortcomings, we propose in this paragraph a simple solution which is based on the introduction of a new (internal) action, called the generic temporal violation and noted $\pi$.

We furthermore extend the suffix of an observable action, using notation $a\{t, Q\}$, in order to specify explicitly the process $Q$ to be executed when action $a$ cannot be realized during time t. This simple extension, is formalized in table 3, where only the semantic rules which have been added and modified with respect to table 2 are detailed. This extension presents the following characteristics:

1. the exception process $Q$ is specified at the level of each relevant observable action in a specification, without any additional operator, like the previous choice operator

2. the first action of the exception process $Q$ does not need to be prefixed by an artificial delay operator as in the previous solution. This is a direct consequence of rules (2.c) and (7.d) of the operational semantics. A temporal violation of $a\{t, Q\}$ leads to the occurrence of the generic violation action $(\pi)$ which gives the control to process $Q$ (see rule (2.c)). Note however that action $\pi$ has less priority than action $a_{s}$ (see rule (7.d)), which makes it possible to obtain the desired behavior (see in particular requirement 1 stated in paragraph 3.1)

3. $a\{\mathrm{t}\} ; \mathrm{P}$ means that there is no explicit exception process associated with $a$, and consequently it is equivalent to $a\{\mathrm{t}$, stop $\} ; \mathrm{P}$ 
The other rules for the $\pi$ actions are almost straightforward, and, as expected, the occurrence of action $\pi$ does not resolve the choice (see rule (5.c)).

As an illustration, let us consider the same example as the one presented in paragraph 2.3.4, but using now the construction introduced in this paragraph instead of the choice operator:

Medium $=\mathrm{a} ; \Delta^{\mathrm{m}} \Omega^{\mathrm{M}-\mathrm{m}} \mathrm{b}\{\mathrm{M}-\mathrm{m}$, error; stop $\} ;$ Medium

We believe that the proposed solution is very simple, as it implies a very simple extension of the syntactic model, as well as the introduction of only one special purpose action in the semantic model, action $\pi$. However, it does not completely resolve shortcomings 2 and 3 identified previously.

\begin{tabular}{|c|c|c|c|}
\hline$(2 . a)$ & $a\{u, Q\} ; P \stackrel{a_{s}}{\rightarrow} P(a \in A)$ & $\begin{array}{l}(2 . b) \\
(2 . c)\end{array}$ & $\begin{array}{l}a\{u+t, Q\} ; P \stackrel{t}{\rightarrow} a\{u, Q\} ; P(a \in A) \\
a\{0, Q\} ; P \stackrel{\pi}{\rightarrow} Q(a \in A)\end{array}$ \\
\hline$(4 d)$ & $P \stackrel{\pi}{\rightarrow} P^{\prime}$ & & $P \stackrel{\pi}{\rightarrow} P^{\prime}$ \\
\hline$(4 . d)$ & $\overline{\Delta^{0} P \stackrel{\pi}{\rightarrow} P^{\prime}}$ & $\left(4^{\prime} . e\right)$ & $\overline{\Omega^{u} P \stackrel{\pi}{\rightarrow} \Omega^{u} P^{\prime}}$ \\
\hline$(5-1)$ & $P \stackrel{\pi}{\rightarrow} P^{\prime}$ & $(f-d)$ & $P \stackrel{\pi}{\rightarrow} P^{\prime}$ \\
\hline$(\partial . c)$ & $\overline{P[] Q \stackrel{\pi}{\rightarrow} P^{\prime}[] Q}$ & $(0 . a)$ & $\overline{P|[L]| Q \stackrel{\pi}{\rightarrow} P^{\prime}|[L]| Q}$ \\
\hline & $P \stackrel{\pi}{\rightarrow} P^{\prime} \quad P \stackrel{a_{g}}{\rightarrow} \quad \forall a \in L$ & $(8 d)$ & $P \stackrel{\pi}{\rightarrow} P^{\prime}$ \\
\hline$($ (I.a) & $\overline{\text { hide } L \text { in } P \stackrel{\pi}{\rightarrow} \text { hide } L \text { in } P^{\prime}}$ & (8.d) & $\overline{P \gg Q \stackrel{\pi}{\rightarrow} P^{\prime} \gg Q}$ \\
\hline & $P \stackrel{\pi}{\rightarrow} P^{\prime}$ & $(0 f)$ & $Q \stackrel{\pi}{\rightarrow} Q^{\prime}$ \\
\hline (9.d) & $\overline{P\left[>Q \stackrel{\pi}{\rightarrow} P^{\prime}[>Q\right.}$ & (9.J) & $\overline{P\left[>Q \stackrel{\pi}{\rightarrow} P\left[>Q^{\prime}\right.\right.}$ \\
\hline$(10 . a)$ & $\frac{E\left[a_{1} / a_{1}^{\prime} \cdots a_{n} / a_{n}^{\prime}\right] \stackrel{g}{\rightarrow} E^{\prime} \quad P\left[a_{1}^{\prime} \cdots a_{n}^{\prime}\right]:=E}{P\left[a_{1} \cdots a_{n}\right] \stackrel{g}{\rightarrow} E^{\prime}}$ & $(g$ & $\left.\left\{a_{s}, a_{w} \mid a \in A^{i, \delta}\right\} \cup D^{\infty} \cup\{\pi\}\right)$ \\
\hline
\end{tabular}

Table 3: Temporal violation action $\pi$

\subsection{The temporal disrupt operator}

Resolving completely the previous shortcomings means that one may desire to express the exception process in a way which is completely disjoint of the specification of the observable actions. Following such an approach emphasizes a modular style of specification, the specification of the exception behaviors being described completely independently of the core specification.

To do so, our concept of temporal violation is extended in order to maintain the knowledge about what action was not able to be executed within its specific time interval. Thus, for any observable action $a$ in the syntactic model, we introduce three special actions:

1. $a^{0}$ characterizes an inevitable temporal violation (i.e. which is independent of the behavior of the environment)

2. $a^{1}$ characterizes a potential temporal violation (i.e. which may either be avoided or be transformed into an inevitable temporal violation depending on the behavior of the environment)

3. $\sigma^{a}$ characterizes the fact that a potential temporal violation has been treated by an exception behavior

Furthermore, we introduce action $\sigma$ for characterizing that an inevitable temporal violation has been treated by an exception behavior.

We still use notation $a\{t, Q\}$ for specifying the exception behavior associated locally to action $a$, as defined in the previous paragraph. In the solution proposed here, there exists furthermore the possibility of using the temporal disrupt operator for specifying (more globally) the exception process to be associated with an action or a set of actions. In the proposal formalized in table 4, both constructs may cohabit. As seen below, they do not have exactly the same meaning, as the first one has only a local scope and the other one may have a more global scope, depending on where the temporal disrupt operator is located in the specification. 


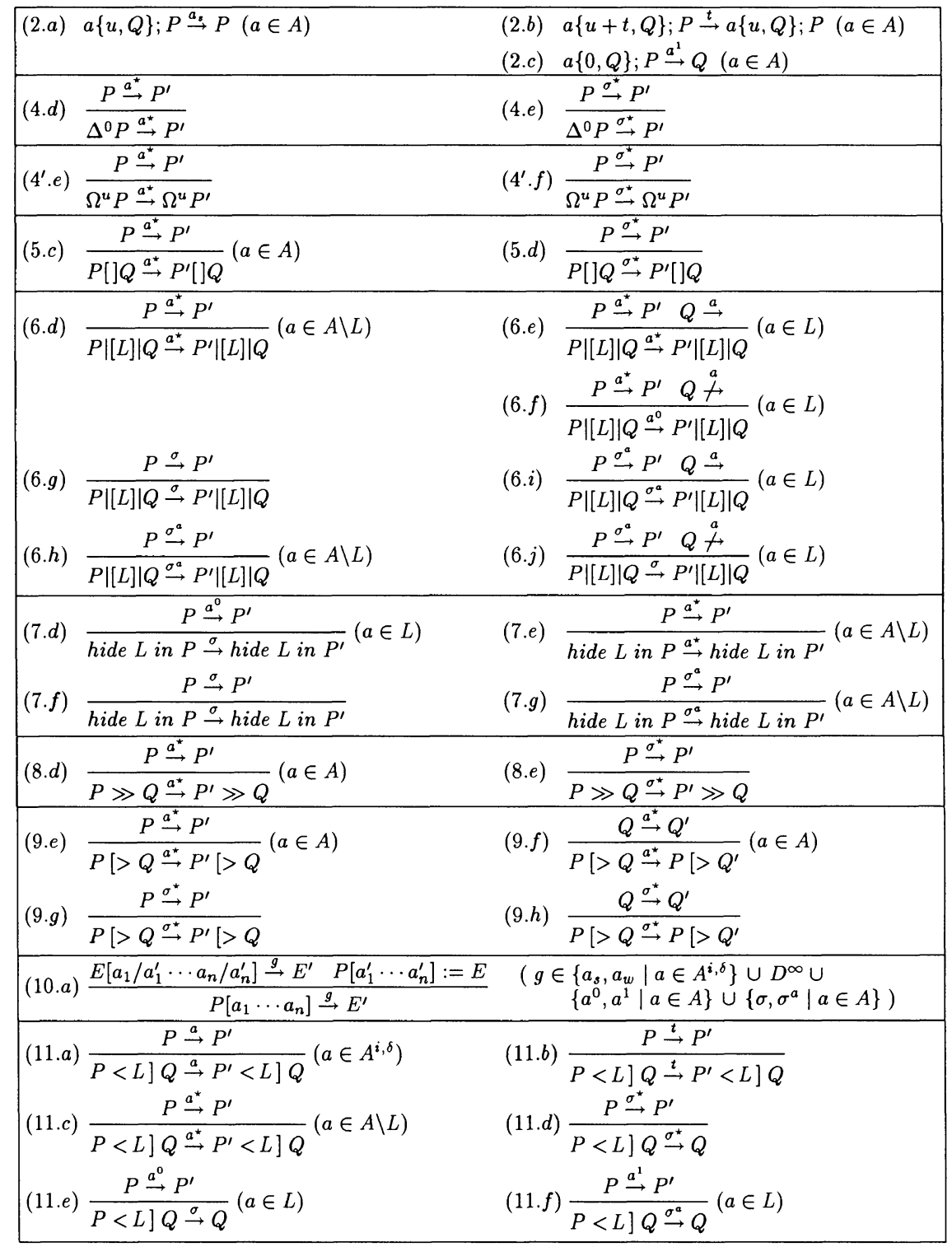

Table 4: Temporal violation actions $\mathrm{a}^{0}$ and $\mathrm{a}^{1}$ 
For instance, let us assume:

$\mathrm{S} 1=a\{0, \mathrm{Q}\} ; \mathrm{P} \|[\mathrm{a}] \mid \mathrm{E}$

$\mathrm{S} 2=(a\{0, \mathrm{Q} 1\} ; \mathrm{P}|[\mathrm{a}]| \mathrm{E})<\mathrm{a}] \mathrm{Q} 2$

In case of a temporal violation for action $a$, specification $\mathrm{S} 1$ transforms itself in $\mathrm{Q}|[\mathrm{a}]| \mathrm{E}$, whereas specification $\mathrm{S} 2$ transforms itself in Q2.

In the sequel we provide some explanations of the inference rules detailed in table 4 :

1. $a^{*}$ stands for either $a^{0}$ or $a^{1}$

2. $\sigma^{*}$ stands for either any $\sigma^{a}$ with $a \in A$ or $\sigma$

3. rule (2.c) generates a potential temporal violation $a^{1}$ when the time interval reaches its upper bound, in a way similar to the $\pi$ action defined in the previous paragraph

4. rule (6.f) generates an inevitable temporal violation $a^{0}$ when the environment cannot offer an action $a$ to be synchronized with a potential temporal violation $a^{1}$

5. rule (11.f) transforms potential temporal violation $a^{1}$ into $\sigma^{a}$ action (case of the temporal disrupt operator)

6. rule (11.e) transforms inevitable temporal violation $a^{0}$ into $\sigma$ action (case of the temporal disrupt operator)

7. rule (7.d) transforms inevitable temporal violation $a^{0}$ into $\sigma$ action (case of the hide operator)

8. rule (6.j) generates a $\sigma$ action when the environment cannot offer an action $a$ to be synchronized with a $\sigma^{a}$ action

\section{Temporal bisimulations}

In this section we consider only the basic model extended with the latency operator, whose complete operational semantics has been provided in table 2 . Our aim is to define a strong and a weak equivalence and to derive some interesting properties of the model.

\subsection{Strong Equivalence}

Definition 1 For $a \in A^{i, \delta}$ and $t \in D^{\infty}$ :

1. $P \stackrel{a_{5}}{\mapsto} P^{\prime}$ means $P \stackrel{a_{s}}{\rightarrow} P^{\prime}$

2. $P \stackrel{a_{w}}{\longrightarrow} P^{\prime}$ means $P \stackrel{a_{w}}{\longrightarrow} P^{\prime}$

3. $P \stackrel{t}{\mapsto} P^{\prime}$ means $P \stackrel{t_{1}}{\rightarrow} \stackrel{t_{2}}{\rightarrow} \cdots \stackrel{t_{n}}{\rightarrow} P^{\prime}$ and $t=t_{1}+t_{2}+\cdots+t_{n}$

Definition 2 (strong bisimulation) A binary relation $\mathcal{S} \subseteq \mathcal{P} \times \mathcal{P}$ is a strong bisimulation if $(P, Q) \in \mathcal{S}$ implies, for all $g \in\left\{a_{s}, a_{w} \mid a \in A^{i, \delta}\right\} \cup D^{\infty}$,

1. if $P \stackrel{g}{\rightarrow} P^{\prime}$, then $\exists Q^{\prime}$ such that $Q \stackrel{g}{\rightarrow} Q^{\prime}$ and $\left(P^{\prime}, Q^{\prime}\right) \in \mathcal{S}$

2. if $Q \stackrel{g}{\rightarrow} Q^{\prime}$, then $\exists P^{\prime}$ such that $P \stackrel{g}{\mapsto} P^{\prime}$ and $\left(P^{\prime}, Q^{\prime}\right) \in \mathcal{S}$

Definition 3 (strong equivalence) $P$ and $Q$ are strongly equivalent, denoted $P \sim Q$, if there exists a strong bisimulation $\mathcal{R}$ such that $(P, Q) \in \mathcal{R}$.

\section{Proposition 4}

1. $\sim$ is the largest strong bisimulation.

$2 . \sim$ is an equivalence relation.

The proposition below characterizes the relationship between the delay operator $\left(\Delta^{t}\right)$, the latency operator $\left(\Omega^{\mathrm{t}}\right)$, as well as the $i\{t\}$ construct. Note, in particular, that proposition 8.6 shows that the $i\{t\}$ construct is not required in our model, as previously already mentioned.

\section{Proposition 5}
1. $\Delta^{t} \Delta^{u} P \sim \Delta^{t+u} P$
3. $\Delta^{t} \Omega^{u} P \sim \Omega^{t+u} \Delta^{t} P$
5. $\Delta^{0} P \sim \Omega^{0} P \sim P$
2. $\Omega^{t} \Omega^{u} P \sim \Omega^{\max (t, u)} P$
4. $\Omega^{t} \Delta^{u} P \sim \Delta^{u} \Omega^{\max (0, t-u)} P$
6. $i\{t\} ; P \sim\left(\Omega^{t}\right.$ exit $) \gg P$ 
The proposition below characterizes the relationship of our temporal operators, $\Delta^{t}$ and $\Omega^{t}$, with the others operators of Basic LOTOS.

\section{Proposition 6}
1. $\Delta^{t}(P[] Q) \sim \Delta^{t} P[] \Delta^{t} Q$
1'. $\Omega^{t}(P[] Q) \sim \Omega^{t} P[] \Omega^{t} Q$
2. $\Delta^{t}(P|[\mathrm{~L}]| Q) \sim \Delta^{t} P|[\mathrm{~L}]| \Delta^{t} Q$
2'. $\Omega^{t}(P|[\mathrm{~L}]| Q) \sim \Omega^{t} P|[\mathrm{~L}]| \Omega^{t} Q$
3. $\Delta^{t}$ (hide $L$ in $P$ ) $\sim$ hide $L$ in $\Delta^{t} P$
4. $\Delta^{t}(P \gg Q) \sim \Delta^{t} P \gg Q$
5. $\Delta^{t}\left(P[>Q) \sim \Delta^{t} P\left[>\Delta^{t} Q\right.\right.$
5. $\Omega^{t}\left(P[>Q) \sim \Omega^{t} P\left[>\Omega^{t} Q\right.\right.$

The proposition below states that $\sim$ is a congruence.

Proposition 7 Let $P \sim Q$. Then
1. $\Delta^{t} P \sim \Delta^{t} Q$
3. $a\{t\} ; P \sim a\{t\} ; Q$, for all $a \in A^{i}$
2. $\Omega^{t} P \sim \Omega^{t} Q$
5. $P|[\mathrm{~L}]| R \sim Q|[\mathrm{~L}]| R$
4. $P[] R \sim Q[] R$
7. $P \gg R \sim Q \gg R$
6. hide $L$ in $P \sim$ hide $L$ in $Q$
9. $P[>R \sim Q[>R$
8. $R \gg P \sim R \gg Q$
10. $R[>P \sim R[>Q$

\subsection{Weak Equivalence}

Definition 4 For $a \in A^{\delta}$ and $t \in D^{\infty}$ :

1. $P \stackrel{a_{s}}{\Rightarrow} P^{\prime}$ means $P\left(\stackrel{i_{s}}{\rightarrow}\right)^{*} \stackrel{a_{3}}{\rightarrow}\left(\stackrel{i_{s}}{\rightarrow}\right)^{*} P^{\prime}$

2. $P \stackrel{a_{w}}{\Rightarrow} P^{\prime}$ means $P \stackrel{i_{x_{1}}}{\longrightarrow} \cdots \stackrel{i_{x_{k-1}}}{\longrightarrow} \stackrel{a_{x_{k}}}{\longrightarrow} \stackrel{i_{x_{k+1}}}{\longrightarrow} \ldots \stackrel{i_{x_{n}}}{\longrightarrow} P^{\prime}$ and $\bigwedge_{1 \leq k \leq n} x_{k}=w(s=1, w=0$ in $\wedge)$

3. $P \stackrel{i s}{\Rightarrow} P^{\prime}$ means $P(\stackrel{i s}{\rightarrow})^{*} P^{\prime}$

4. $P \stackrel{i_{y}}{\Rightarrow} P^{\prime}$ means $P \equiv P^{\prime}$ or $P \stackrel{i_{x_{1}}}{\longrightarrow} \cdots \stackrel{i_{x_{n}}}{\longrightarrow}$ and $\bigwedge_{1 \leq k \leq n} x_{k}=w(s=1, w=0$ in $\wedge)$

5. $P \stackrel{t}{\Rightarrow} P^{\prime}$ means $P\left(\stackrel{i_{s}}{\rightarrow}\right)^{*} \stackrel{t_{1}}{\rightarrow}\left(\stackrel{i_{s}}{\rightarrow}\right)^{*} \stackrel{t_{2}}{\rightarrow} \cdots\left(\stackrel{i_{s}}{\rightarrow}\right)^{*} \stackrel{t_{n}}{\rightarrow}\left(\stackrel{i_{s}}{\rightarrow}\right)^{*} P^{\prime}$ and $t=t_{1}+t_{2}+\cdots+t_{n}$

Definition 5 (weak bisimulation) A binary relation $\mathcal{S} \subseteq \mathcal{P} \times \mathcal{P}$ is a weak bisimulation if $(P, Q) \in \mathcal{S}$ implies, for all $g \in\left\{a_{s}, a_{w} \mid a \in A^{i, \delta}\right\} \cup D^{\infty}$,

1. if $P \stackrel{g}{\rightarrow} P^{\prime}$, then $\exists Q^{\prime}$ such that $Q \stackrel{g}{\Rightarrow} Q^{\prime}$ and $\left(P^{\prime}, Q^{\prime}\right) \in \mathcal{S}$

2. if $Q \stackrel{g}{\rightarrow} Q^{\prime}$, then $\exists P^{\prime}$ such that $P \stackrel{g}{\Rightarrow} P^{\prime}$ and $\left(P^{\prime}, Q^{\prime}\right) \in \mathcal{S}$

Definition 6 (weak equivalence) $P$ and $Q$ are weakly equivalent, denoted $P \approx Q$, if there exists a weak bisimulation $\mathcal{R}$ such that $(P, Q) \in \mathcal{R}$.

\section{Proposition 8}

$1 . \approx$ is the largest weak bisimulation.

2 . $\approx$ is an equivalence relation.

\section{Proposition 9}

1. If $\mathrm{P}^{\stackrel{t}{\rightarrow}} \mathrm{P}^{\prime}, \mathrm{Q} \stackrel{t}{\rightarrow} \mathrm{Q}^{\prime}$ and $\mathrm{P} \approx \mathrm{Q}$, then $\mathrm{P}^{\prime} \approx \mathrm{Q}^{\prime}$.

2. If $\mathrm{P}^{\rightarrow} \stackrel{t}{\rightarrow} \mathrm{P}^{\prime}, \mathrm{Q}(\stackrel{i \rightarrow}{\rightarrow})^{*} \mathrm{Q}^{\prime}$ and $\mathrm{P} \approx \mathrm{Q}$, then $\mathrm{P} \approx \mathrm{Q}^{\prime}$.

The proposition below states that $\approx$ is a congruence for all the operators, with the exception of the choice operator, as in Basic LOTOS.

\section{Proposition 10 Let $P \approx Q$. Then}
1. $\Delta^{t} P \approx \Delta^{t} Q$
3. $a\{t\} ; P \approx a\{t\} ; Q$, for all $a \in A^{i}$
2. $\Omega^{t} P \approx \Omega^{t} Q$
4. $P|[\mathrm{~L}]| R \approx Q|[\mathrm{~L}]| R$
6. $P \gg R \approx Q \gg R$
5. hide $L$ in $P \approx$ hide $L$ in $Q$
8. $P[>R \approx Q[>R$
7. $R \gg P \approx R \gg Q$ 
Proof: We show only the case of the hide operator. Let

$$
\mathcal{S}=\{(\text { hide } \mathrm{L} \text { in } \mathrm{P} \text {, hide } \mathrm{L} \text { in } \mathrm{Q}) \mid \mathrm{P}, \mathrm{Q} \in \mathcal{P} \text { and } \mathrm{P} \approx \mathrm{Q}\}
$$

It is sufficient to show that whenever hide $\mathrm{L}$ in $\mathrm{P} \stackrel{g}{\rightarrow}$ hide $\mathrm{L}$ in $\mathrm{P}^{\prime}$, then hide $\mathrm{L}$ in $\mathrm{Q} \stackrel{g}{\Rightarrow}$ hide $\mathrm{L}$ in $\mathrm{Q}^{\prime}$ for some $\mathrm{Q}^{\prime}$ and ( hide $\mathrm{L}$ in $\mathrm{P}^{\prime}$, hide $\mathrm{L}$ in $\mathrm{Q}$ ') $\in \mathcal{S}$.

1. Assume hide $\mathrm{L}$ in $\mathrm{P} \stackrel{t}{\rightarrow}$ hide $\mathrm{L}$ in $\mathrm{P}^{\prime}$ for $t>0$. We must have $\mathrm{P} \stackrel{t}{\rightarrow} \mathrm{P}^{\prime}$ and, since $\mathrm{P} \approx \mathrm{Q}$, there exists $Q^{\prime}$ such that $\mathrm{Q}^{t} \Rightarrow \mathrm{Q}^{\prime}$ and $\mathrm{P}^{\prime} \approx \mathrm{Q}^{\prime}$. Assume that $\mathrm{Q} \stackrel{t}{\Rightarrow} \mathrm{Q}^{\prime}$ is achieved from the following sequence,

$$
\mathrm{Q} \equiv \mathrm{Q}_{0}^{\prime}\left(\stackrel{i_{s}}{\rightarrow}\right)^{*} \mathrm{Q}_{1} \stackrel{t_{1}}{\rightarrow} \mathrm{Q}_{1}^{\prime}\left(\stackrel{i_{s}}{\rightarrow}\right)^{*} \mathrm{Q}_{2} \cdots \mathrm{Q}_{n-1}^{\prime}\left(\stackrel{i_{s}}{\rightarrow}\right)^{*} \mathrm{Q}_{n} \stackrel{t_{n}}{\rightarrow} \mathrm{Q}_{n}^{\prime}\left(\stackrel{i_{s}}{\rightarrow}\right)^{*} \mathrm{Q}_{n+1} \equiv \mathrm{Q}^{\prime}
$$

where $t=t_{1}+t_{2}+\cdots+t_{n}$.

By $\mathrm{P} \stackrel{t}{\rightarrow} \mathrm{P}^{\prime}$ and the time continuity property,

$$
\mathrm{P} \equiv \mathrm{P}_{1} \stackrel{t_{1}}{\rightarrow} \mathrm{P}_{2} \cdots \mathrm{P}_{n} \stackrel{t_{n}}{\rightarrow} \mathrm{P}_{n+1} \equiv \mathrm{P}^{\prime}
$$

By rule (7.a) of table 2, we can conclude that

$$
\text { hide } \mathrm{L} \text { in } \mathrm{Q}^{\prime}{ }_{k}\left(\stackrel{i_{9}}{\rightarrow}\right)^{*} \text { hide } \mathrm{L} \text { in } \mathrm{Q}_{k+1} \text {, for all } k \in\{0 \ldots n\}
$$

It remains to prove that

$$
\text { hide } \mathrm{L} \text { in } \mathrm{Q}_{k} \stackrel{t_{k}}{\rightarrow} \text { hide } \mathrm{L} \text { in } \mathrm{Q}_{k}{ }_{k} \text {, for all } k \in\{1 \ldots n\}
$$

By rule (7.b), it is sufficient to prove that

$$
\mathrm{Q}_{k} \stackrel{a_{s}}{\dagger} \text {, for all } a \in L \text { and all } k \in\{1 \ldots n\}
$$

Let us assume, by absurd, that $\mathrm{Q}_{k} \stackrel{a_{3}}{\rightarrow}$, for some $a \in L$. Using the properties of proposition 9 , we can establish that

$$
\mathrm{P}_{k} \approx \mathrm{Q}_{k} \text { and } \mathrm{P}_{k} \approx \mathrm{Q}_{k-1}^{\prime} \text {, for all } k \in\{1 \ldots n\}
$$

So $\mathrm{P}_{k} \stackrel{a_{s}}{\Rightarrow}$, but, by the maximal progress property, we will have $\mathrm{P}_{k} \stackrel{a_{s}}{\rightarrow}\left(\stackrel{i_{s}}{\rightarrow}\right)^{*}$. However, due to the initial hypothesis, we can easily establish that $\mathrm{P}_{k} \stackrel{a}{\rightarrow}$, for all $a \in L$ and all $k \in\{1 \ldots n\}$

2. the case hide $\mathrm{L}$ in $\mathrm{P} \stackrel{g}{\rightarrow}$ hide $\mathrm{L}$ in $\mathrm{P}^{\prime}$ for $g \in\left\{a_{s}, a_{w} \mid a \in A^{i, \delta}\right\}$ is straightforward.

Note that, with the proposed equivalence, behaviors $\Omega^{t}$ a;stop and a;stop (with $t>0$ ) are distinguished, as well as $\mathrm{i}\{\mathrm{t}\} ; \mathrm{a}$;stop and a;stop (with $t>0$ ), which seems to be not the case for the weak equivalence proposed in [LL94]. This latter point leads to potential problems as far as the congruence with respect to the hide is concerned, as it may be illustrated by the following example:

Assuming $a ; b\{0\} ;$ stop $\approx \mathrm{i}\{\mathrm{t}\} ; \mathrm{a} ; \mathrm{b}\{0\} ;$ stop, then it may be shown than hide $\mathrm{a}$ in $\mathrm{a} ; \mathrm{b}\{0\}$;stop $\not$ hide a in $\mathrm{i}\{\mathrm{t}\} ; \mathrm{a} ; \mathrm{b}\{0\} ; \mathrm{stop}$, as a time progression in the first process leads to process stop, whereas it may lead to the occurrence of action $b$ in the second process.

With the proposed equivalence, $\mathrm{i}\{\mathrm{t}\} ; \mathrm{a} ;$ stop $\not \approx$ a;stop (and consequently a;b $\{0\}$;stop $\not \approx$ $\mathrm{i}\{\mathrm{t}\} ; \mathrm{a} ; \mathrm{b}\{0\} ;$ stop), as action $\mathrm{a}_{s}$ in $\mathrm{a} ;$ stop cannot be simulated by any action sequence of $\mathrm{i}\{\mathrm{t}\} ; \mathrm{a} ; \mathrm{stop}$.

\section{Conclusion}

Starting from an earlier experience with the design of RT-LOTOS [CdCS93] and taking into account the proposals of G. Leduc, namely Timed LOTOS [LL92] and ET-LOTOS [LL93b], three main contributions have been provided in the paper:

1. the definition of the latency operator

2. the proposal of different alternatives for expressing temporal exception handling

3. the definition of a weak equivalence for the basic model with time nondeterminism, which is a congruence for the hide and enabling operators

The latency operator provides several advantages with respect to others proposals for expressing time nondeterminism. In particular: 
1. no additional internal action is introduced for simulating the time nondeterminism, as the semantical observable actions carry directly this information (i.e. the time nondeterminism)

2. the latency operator permits to express both an urgent hide (like in Timed LOTOS and ET-LOTOS) and a non urgent hide (like in RT-LOTOS)

3. the maximal progress property is satisfied, with respect to the strong internal action

4. it is possible to define a weak equivalence which is a congruence for the hide and enabling operators

The basic model with time nondeterminism can be extended in several ways, by introducing for instance :

1. one of the proposals made for expressing temporal exception behaviors

2. the $a\{\mathrm{t}\} @ \mathrm{u}$ construct of ET-LOTOS

3. a nondeterministic delay operator $\Delta^{t, u} \mathrm{P}$ which could be defined as $\Delta^{t} \Omega^{u-t} \mathrm{P}$

\section{Acknowledgements}

The authors would like to thank Guy Leduc for interesting discussions and anonymous referees for helpful comments on this paper.

\section{References}

[BB87] T. Bolognesi and E. Brinksma. Introduction to the ISO Specification Language LOTOS. Computer Networks and ISDN Systems, 14(1):25-59, 1987.

[BL92] T. Bolognesi and F. Lucidi. LOTOS-like process algebras with urgent or timed interactions. In FORTE'91, pages 249-264. IFIP, North-Holland, 1992.

[CdCS93] J.P. Courtiat, M.S. de Camargo, and D.E. Saidouni. RT-LOTOS: LOTOS temporisé pour la spécification de systèmes temps réel. In R. Dssouli, G.v. Bochmann, and L. Lévesque, editors, Ingénierie des Protocoles CFIP'93, pages 427-441, Hermès, Paris, 1993.

[LL92] G. Leduc and L. Léonard. A timed LOTOS supporting a dense time domain and including new timed operators. In Proc. Fifth Intern. Confer. on Formal Description Techniques (FORTE), Lannion, France, October 1992.

[LL93a] G. Leduc and L. Léonard. Comment rendre LOTOS apte à spécifier des systèmes temps réel ? In R. Dssouli, G.v. Bochmann, and L. Lévesque, editors, Ingénierie des Protocoles CFIP'93, pages 407-425, Hermès, Paris, 1993.

[LL93b] L. Léonard and G. Leduc. An enhanced version of timed LOTOS and its application to a case study. In Proc. Sixth Intern. Confer. on Formal Description Techniques (FORTE), Boston, Massachusetts, October 1993.

[LL94] G. Leduc and L. Léonard. A formal definition of time in LOTOS. Technical report, Université de Liège, Belgium, 1994.

[NS91] X. Nicollin and J. Sifakis. An overview and synthesis on timed process algebras. In $R E X$ Workshop "Real-Time: Theory in Practice", volume 600 of LNCS, pages 526-548. SpringerVerlag, 1991. 